International Journal of Social Science and Economic Research

ISSN: $2455-8834$

Volume:06, Issue:05 "May 2021"

\title{
LOST IN TRANSLATION: EXPLORING EUROCENTRICISM IN KEY DIAGNOSTIC TOOLS
}

\author{
Sairah Mamik \\ Vasant Valley school \\ DOI: 10.46609/IJSSER.2021.v06i05.017 URL: https://doi.org/10.46609/IJSSER.2021.v06i05.017
}

\begin{abstract}
Intelligence tests are scientifically devised tools that are used to identify disabilities, account for gaps in potentiality and performance in school, and assess eligibility for welfare rehabilitation, and guidance. The Wechsler Intelligence Scale for Children (WISC) is a popular intelligence test in English for children between the ages of 6 and 16, which was subsequently adapted for Indian students by Arthur J. Malin to create the Malin's Intelligence Scale for Indian Children. This paper has explored challenges associated with the reliability, validity, and dependability of the MISIC as an intelligence test in India. It has been found that the MISIC neither accounts for cultural and educational differences amongst students in India nor has it been renewed since its initial publication to account for the Flynn Effect. Translating the test to indigenous languages is a cumbersome task that further burdens overstretched clinicians and educators in the public sector of India. Lastly, this paper identifies structural problems that restrict psychiatric diagnoses in less developed regions of India and identifies the need for modernizing and improving statesponsored mechanisms that seek to implement the same.
\end{abstract}

Keywords: education, children, WISC, MISIC, language

\section{Introduction}

The Wechsler Intelligence Scale for Children (WISC) is an intelligence test for children between the ages of 6 and 16. It forms a part of the Wechsler intelligence scale system, which is used to test intelligence levels and mental functioning for individuals of different age groups (preschoolers, adults, and so on). Along with other Wechelsar Scale Tests, the WISC is revised to account for the Flynn Effect (explained later). Currently, the most recent and fifth version of the WISC is in use. The test, which takes 45-65 minutes to administer, is used to determine the Full-Scale IQ of a child, which is considered to be representative of their general intellectual ability (Ward, 1995). Additionally, it is also used to determine the child's abilities in discrete cognitive domains which are measured through these five primary index scores: the Verbal 


\section{International Journal of Social Science and Economic Research}

ISSN: $2455-8834$

Volume:06, Issue:05 "May 2021"

Comprehension Index (a measure of verbal concept formation), the Visual-Spatial Index (a measure of visual-spatial processing), the Fluid Reasoning Index (a measure of inductive and quantitative reasoning), the Working Memory Index (a measure of working memory ability), and the Processing Speed Index (a measure of processing speed). Recent revisions of the Wechsler Intelligence System also provides for several complementary subtests (such as the Quantitative Reasoning Index, the Auditory Working Memory Index, the Nonverbal Index, the General Ability Index, etc.) that yield complementary composite scores measuring more specific cognitive abilities which can in-turn helps in diagnosing and identifying specific learning disabilities, particularly dyslexia and dyscalculia. The assessment uses 2 subtests to identify each of the primary index scores, hence creating a total of 10 primary subtests. In total, the WISC-V has a total of 21 subtests, yielding 15 composite scores (Wechsler, 2015).

Since its development in 1949, it has been used not only as an intelligence test in academic and professional spaces, but also as a diagnostic tool, and has emerged as an important tool in educational psychology. Even though research suggests that it cannot exclusively be used as a tool for the detection of ADHD and other specific learning disorders (IQ cannot be used exclusively to diagnose learning problems), it does rule out certain other problems that could have affected learning and performance in addition to it forming an important part of the multitest battery that is administered to children (Watkins, 1997). It continues to be important in the context of educational policy, teaching, and general development because of its ability to measure discrepancies between intelligence and academic performance, to contrast cognitive development with chronological age, and get an insight into intellectual giftedness, learning difficulties, and cognitive strengths and weaknesses of students. Research also suggests that the WISC-V is connected to other important measures of achievement, adaptive behavior, executive function, and behavior and emotion (Kaplan \& Saccuzzo, 2009). The WISC does away with many criticisms of the usage of IQ as a test of intelligence and mental functioning by providing diverse test results which can be analyzed to arrive at more holistic and comprehensive conclusions in comparison to a single IQ score. When used in conjunction with an achievement test (such as the Woodcock-Johnson III), it can be used to diagnose learning difficulties by allowing for a comparison between actual achievement scores and expected levels of achievement for that intelligence level (Kaplan \& Saccuzzo, 2009).

\section{Background}

The WISC emerged as one of the most holistic and important measures of intelligence and mental functioning, as well as a useful supplementary tool in the diagnosis of learning difficulties. Whenever a test is applied in any community which does not have similar development levels and cultural norms, it tends to be ineffective. Hence translating a test to 


\section{International Journal of Social Science and Economic Research}

ISSN: $2455-8834$

Volume:06, Issue:05 "May 2021"

another language is not necessarily enough for it to be applied in other countries and communities, it must be effectively contextualized according to children there.

Because of its utility as an academic and clinical psychological tool, it has been translated or adapted to many languages including Spanish, Portuguese, Arabic, Icelandic, Norwegian, Swedish, and many more. To facilitate smoother administration of these tests in languages other than English, norms have been established for administration in a number of countries including Brazil, Portugal, France, Canada, Germany, Austria, and Switzerland. Countries including the United States, Canada, United Kingdom, and Australia have their norms as well, with revisions of norms being done with each translation and at the release of every new edition (Shyam \& Khan, 2009).

The discourse surrounding mental health, and especially its correlation to mental functioning and academic performance is fairly recent in India, despite mental health care being guaranteed to Indian citizens under the law (Duffy \& Kelley, 2020). Discourse, awareness, human resource development, and financial allocations pertaining to mental illnesses in the country continue to be low (Pailwal, 2019). This particularly holds when it comes to awareness, diagnosis, and treatment of learning disorders and difficulties. The lack of attention and resources devoted to understanding mental functioning amongst Indian students has led to several structural problems, most important of which surrounds an education system that is exclusionary to non-conforming learners. Whilst more modern ideas surrounding holistic education and development have been introduced in India through international models of curriculum, this positive change is restricted to elite, private schools in the most developed cities of the country (which are inaccessible to the vast majority of Indian students). Academic scores have historically been considered as the only important indicator of mental functioning and intelligence, hence negating the need to develop an indigenous test for students that determines mental functioning. Similar to many aspects of Indian education, popular metrics used to determine the intelligence of Indian students are linked to India's colonial past, and more importantly its colonial hangover as eurocentric (and often outdated) practices incompatible with the Indian context continues to be rampant in education systems accessed by most Indians (Sharma, 2014).

Arthur J. Malin developed the Malin's Intelligence Scale for Indian Children (MISIC) by contextualizing the WISC to the educational standards of India and the average intelligence levels of Indian children. MISIC is used to measure intelligence levels in students aged 5-15 by seeking to measure parameters such as comprehension, coding, arithmetic, picture completion, vocabulary, and so on. MISIC is the most widely used intelligence assessment tool in India, despite problems pertaining to the language barrier, a eurocentric approach to intelligence, and the absence of updates to the norms of MISIC over the past few decades. 
International Journal of Social Science and Economic Research

ISSN: $2455-8834$

Volume:06, Issue:05 "May 2021"

\section{Discussion}

Adapting and implementing the Wechsler Intelligence Scale for Children for students in India to the Malin's Intelligence Scale for Indian Children has been challenging, primarily due to differences in culture, language, intelligence levels and definitions, and the education system. Malin acknowledged the impact of culture and intelligence levels and justified adopting a foreign test for Indian children instead of developing an indigenous one by emphasizing the need for Indian clinical psychology to adopt the latest advancements in psychometrics promptly (Shyam \& Khan, 2009). Implementing the scale in India has proven to be practically difficult and cumbersome for clinical practitioners. The MISIC is designed in English, which is not fluently spoken by a significant section of Indian children, especially those that belong to socioeconomically marginalized communities and families. Hence the cumbersome responsibility of translating this test to local languages (many of which exist due to the diverse cultures in India) falls on clinicians, many of whom form a part of the neglected public mental health care infrastructure of India. Secondly, the test is blind and ignorant of the diverse demographic structure of India and provides no linkages between the socio-economic background of students and their performance (a shortcoming common to many intelligence tests). More importantly, the norms of MISIC are outdated which causes this test to be less efficient due to the Flynn Effect. The Flynn effect is defined as the substantial and long-sustained increase in test scores across the world over the 20th century (Baker, et. al., 2015). Improved performance in tests can be linked to multiple socioeconomic factors which impact an entire generation at the community level, including access to nutrition, the prevalence of infectious diseases, and most importantly, changes in the education system, and the general environment that surrounds students (Flynn, 2009). Improvements in these factors reflect a subsequent increase in intelligence testing scores. To ensure standardization and effective diagnosis, intelligence tests should account for the Flynn Effect through revisions (as explained before the WISC has been revised 4 times to date) Absence of such revisions provides inaccurate results to clinicians, which complicates diagnosis and interventions, and hence makes the MISIC a less effective tool (Plomin, et. al., 2003). This becomes particularly important especially in the context of India and its developing socioeconomic landscape, which necessarily implies that Indian children have undergone numerous significant changes in their intellectual abilities over the past 5 decades which should be accounted for.

Whilst critics in India claim that the application of MISIC is now redundant, some psychometricians suggest that generational changes are minor, which is why the test continues to be used, despite more recent attempts to apply the 4th edition of the WISIC in India (Shyam \& Khan, 2009). These problems, which are rooted in the eurocentric approach adopted by the MISIC make it alien and incompatible with Indian students. This, combined with the academic 


\section{International Journal of Social Science and Economic Research}

ISSN: $2455-8834$

Volume:06, Issue:05 "May 2021"

and health-related importance of having a suitable intelligence test for students points to the need of developing standardized and contextualized methods of testing for psychiatric diagnoses in India.

Academic research and inquiry in India that had been restricted to the adaptation of Western intelligence in the first half of the last century is expanding to develop indigenous models of intelligence testing (Basu, 2016). However, the diversity of language and culture, the lack of standardization of school systems, insufficient economic and human capital investment, and infrastructural inadequacies have emerged as major hindrances to such development. Increased government focus in terms of investment and scrutiny are necessary prerequisites for improving access to reliable intelligence testing. The cultural and academic diversity of India is bound to restrict the reliability of tests, given that reproducing similar results from different regions might be tricky. These differences can be accounted for to create more holistic results in two ways- in the short run clinical psychologists can be trained to contextually account for differences in a standardized manner, and in the long term, state governments can take the initiative to develop tests that are most suitable and appropriate for their school systems. It has also been suggested that an information processing approach should be at the center of future efforts directed at improving intelligence tests in India. This implies that the test should assume a continual pattern of brain development, detaching from the understanding that brain development happens in fixed stages (Rogers, et. al., 1999). It is important to account for the Flynn Effect, which has been vastly understudied and under researched in India, despite the major changes that have occurred in the country's economic, social, cultural, and educational landscape over the past few decades. Setting up research committees and boards that periodically review tests and develop new editions is imperative, which again points to the need for strengthening and modernizing the public infrastructure surrounding mental healthcare.

\section{Conclusion}

Deficiencies in intelligence testing in India are a reflection of the larger gaps in the field of both education and psychology. Despite improvements and attempts at modernization over the past decade, systems governing both education and psychology have been outdated and incompatible with the social context and educational needs of India. Learning differences, for example, continue to be ignored in Indian schools- with curriculum and pedagogy being the same for all students from junior classes. Testing IQ and intelligence levels are important in developing more relevant and dynamic curriculum and assessment tools. These tests also provide key insight in the diagnosis of learning difficulties, which necessitate interventions of their own. Instituting and formalizing such mechanisms is important to develop human capital which is one of the most important determinants of long-term economic growth. Innovations in the field of educational 


\section{International Journal of Social Science and Economic Research}

ISSN: $2455-8834$

Volume:06, Issue:05 "May 2021"

psychology are even more important in the context of the ongoing Covid-19 Pandemic, which has forced students to stay at home and continue their education virtually through online classes. Research suggests that this physical and geographic barrier between educators and students deters academic performance as well as attention and interest levels (Mensah, et. al., 2020). Improving curriculum and pedagogy so that they suit not just virtual mediums but also the mental capacities of students can be instrumental in inducing confidence, increasing participation, and enhancing academic performance (Martin, et. al., 2020).

It is important to consider the potential negative impacts of introducing and popularising intelligence testing. The definition of intelligence continues to be a subject of debate, with different tests focusing on different types of mental functioning. Intelligence testing can sometimes be seen as the single determinant of productivity and even worth, especially in competitive societies such as India where popular conceptions of mental functioning and health are often misguided. Results of these tests should be kept confidential by academic institutions so they can be used to contextualize education without inviting uninformed and premature judgments and evaluations of a young student from the general public. It is equally important for the government to standardized testing and assumes a regulatory and oversight role in this field given that for-profit enterprises have shown the tendency to exploit parent insecurity and the lack of awareness around the implications of intelligence testing to market tests and solutions that are unreliable and unscientific. Socio-economically backward communities in India have largely been excluded from innovations in both mental health and education because of the workings of the free market. Given the importance of education in development and emancipation, and its position as a fundamental right in India, there is an imperative on governments at all levels to attempt to make it more inclusive and accessible to communities that stand to benefit immensely from it. Developing, implementing, and reviewing intelligence testing for underprivileged students is one of the most important ways of actualizing that imperative in the 21 st century.

\section{Bibliography}

Baker, D.P., Eslinger, P.J., Benavides, M., Peters, E., Dieckmann, N.F., and Leon, Juan ( 2015). The cognitive impact of the education revolution: A possible cause of the Flynn Effect on population IQ. Intelligence.

Basu, J. (2016). Present status and challenges of intellectual assessment in India. International Journal of School \& Educational Psychology

Duffy, R.M., and Kelly, B.D. (2020). India's Mental Healthcare Act, 2017: Building Laws, Protecting Rights. Springer Nature. 
International Journal of Social Science and Economic Research

ISSN: 2455-8834

Volume:06, Issue:05 "May 2021"

Flynn, James R. (2009). Requiem for nutrition as the cause of IQ gains: Raven's gains in Britain 1938-2008. Economics and Human Biology

Kaplan, R.M., and Saccuzzo, D.P. (2009). Psychological Testing: Principles, Applications, and Issues (Seventh ed.). Belmont

Martin, C.K., Acal, C., and Hormani, M. (2021). Impact on the Virtual Learning Environment Due to COVID-19. Sustainability

Mensah, E., White, K., Yankey, B., Brown,. S. COVID-19 and Distance Learning: Effects on Georgia State University School of Public Health Students. Frontiers in Public Health

Paliwal, K. (2019). Mental hai': Politicians' pejorative usage of psychiatric terms can't pass off as slang; India staring at big mental health crisis. Firstpost.

Plomin R., DeFries J.C., Craig I.W., and McGuffin P. (2003). Behavioral genetics in the postgenomic era.

Rogers, P.R., Miller, A., and William, Q. (1999). Using information-processing theory to understand planning/Performance relationships in the context of strategy. Strategic Management Journal

Sharma, D.C. (2014). India's new policy aims to close gaps in mental health care. The Lancet.

Shyam, R., and Khan, A.(2009). Psychological Tests Developed for Children in India: A Review of Recent Trends in Research, Practice and Application. Clinical Child Psychology: Contemporary Issues.

Ward, S.B., Ward, T. J., Hatt, C.V., Young, D.L, and Mollner, N.R. (1995). The incidence and utility of the ACID, ACIDS, and SCAD profiles in a referred population. Psychology in the Schools

Watkins, M.W., Kush, J., and Glutting, J.J. (1997). Discriminant and predictive validity of the WISC-III ACID profile among children with learning disabilities Psychology in the Schools 